\title{
Influence of age and purpose for testing on the cut-off selection of serological methods in bovine neosporosis
}

\author{
Gema ÁlvarEZ-GARCÍA ${ }^{\mathrm{a}}$, Esther COLLANTES-FERNÁNDEZ ${ }^{\mathrm{a}}$, \\ Eduardo $\operatorname{COSTAS}^{\mathrm{b}}$, Xabier REBORDOSA $^{\mathrm{c}}$, Luis Miguel ORTEGA-MORA ${ }^{\mathrm{a} *}$ \\ a Departamento de Sanidad Animal, Facultad de Veterinaria, Universidad Complutense de Madrid, \\ Ciudad Universitaria s/n, 28040 Madrid, Spain \\ b Departamento de Producción Animal, Facultad de Veterinaria, Universidad Complutense de Madrid, \\ 28040 Madrid, Spain \\ c Laboratorios HIPRA, S.A., avda. La Selva, 135-17170 Amer (Girona), Spain
}

(Received 26 September 2002; accepted 20 January 2003)

\begin{abstract}
The aim of this study was to investigate the need for different cut-off points, according to animal age and the purpose of testing, for two of the most widely used serological techniques in bovine neosporosis, IFAT and a crude antigen ELISA (Civtest ${ }^{\circledR}$, HIPRA). Therefore, the population reference sera used were defined using a combination of multiple criteria such as epidemiological/ clinical and histopathological parameters and an immunoblot test. Firstly, foetuses and breeding cattle (heifers and cows) were considered as separate subpopulations for serological evaluation. Secondly, cut-off points for each serological technique (IFAT and ELISA) according to age group (foetuses and breeding cattle) and the different practical applications (detection of infection and abortion) were calculated following the receiver operating characteristics (ROC) analysis. Cut-off points were defined, for IFAT and ELISA for aborted breeding cattle and for IFAT alone in the case of the foetuses, assuming an equivalent cost of false positive and negative results. In infected breeding cattle, for IFAT and ELISA and in foetuses for ELISA, two possible cut-off values were obtained, one for a maximum sensitivity and one for a maximum specificity and the intervals of unclear results were defined. In this case, a cut-off value for equal sensitivity and specificity was also estimated. When cut-off points for infected breeding cattle, 1:100-1:250 for IFAT and 0.3060.451 for ELISA were applied to a target population, optimal and similar negative and positive predictive values together with similar apparent and true prevalence results were observed suggesting the possibility of using both tests interchangeably.
\end{abstract}

Neospora caninum / foetus / breeding cattle / serology / cut-off

\section{INTRODUCTION}

Neospora caninum is a cyst-forming coccidian parasite, which has been identified in several domestic species such as cattle, dogs, horses, sheep and goats. Neosporosis has been described as a major cause of abortion in the main cattle producing countries and the infection can also be associated with neonatal mortality and

\footnotetext{
* Correspondence and reprints

Tel.: (34) 91 3943713; fax: (34) 91 3943908; e-mail: luis.ortega@vet.ucm.es
} 
encephalomyelitis in congenitally infected calves [10]. In bovines, different serological methods have been developed to detect parasite specific antibodies [2, 4], primarily the indirect fluorescent antibody test (IFAT) - regarded as a reference test to which other assays have been compared and calibrated with - and different enzymelinked immunosorbent assays (ELISA) [4]. Immunoblotting has been recently used as an aid for other serological tests, rather than as a routine tool for screening cattle sera [2]. Serology in adult cattle enables the individual detection of the infection and, at the herd-level, permits neosporosis to be considered as a cause of reproductive failure by comparing the prevalence of the infection in aborting and non-aborting animals. The presence of specific antibodies in the sera from aborted cows and foetuses is indicative of exposure to Neospora, but examination of the foetus by histological methods is necessary for a definitive diagnosis of abortion due to neosporosis [10].

Serological diagnosis of neosporosis faces several limitations. Foetal age and the time elapsed between infection and abortion are important factors to be considered in the interpretation of foetal serology [35]. In adult cattle, specific antibody levels fluctuate with the animal's age and the state of pregnancy $[8,21,22]$. Therefore, the purposes of serological techniques can be very variable, such as the diagnosis of the infection in foetuses and in cattle that have recently aborted, determination of the infection status in individual cattle i.e. prior to purchase or entry in the breeding herd and estimation of prevalence in epidemiological studies. Because of the different purposes, different cut-off points might be necessary for different applications of testing.

The aim of our study was to investigate the need for different cut-off points, according to animal age and the purpose of testing, for two of the most widely-used serological techniques in bovine neosporosis (IFAT and ELISA). Firstly, serum samples from foetuses and aborted breeding cattle (heifers and cows) were compared in order to determine whether the distribution of serologic values differed in both populations. Secondly, a different cut-off for each serological technique (IFAT and ELISA), age group (foetuses and breeding cattle) and purpose for testing (infection and abortion) were calculated using receiver operating characteristics (ROC). The areas under the ROC curve (AUCs) were calculated for both tests in order to compare their performance. Finally, the influence of the cut-off value applied to detect Neospora infected cattle was studied. True prevalence was estimated in a target population and compared for both IFAT and ELISA and for the different cut-off points obtained and for those recommended in previous works [6, 24].

\section{MATERIALS AND METHODS}

\subsection{Experimental design and serum samples}

In the present work the recommendations given by Greiner and Gardner [13] and Jacobson [16] were carried out in order to calculate the cut-off points for both serological tests, IFAT and ELISA, according to different practical situations. The experimental design was as follows:

Firstly, three different reference populations (groups 1, 2 and 3) and a target population (group 4) were defined:

Group 1: Negative reference sera consisted of two age groups: aborted foetuses $(n=$ 21 ) and breeding cattle (heifers, $n=21$; cows, $n=21$ ). Foetal fluids and serum samples were recovered from each category from animals belonging to herds with no history of reproductive failure caused by Neospora, and a lack of recognition of any tachyzoite immunodominant antigen (IDA) either in foetal fluids or adult sera by an immunoblotting technique [1].

Group 2: Positive reference sera from Neospora aborted foetuses $(n=13)$ and Neospora aborted heifers-cows $(n=33)$. In 
both cases, samples were collected from animals with a Neospora confirmed diagnosis of abortion based on the finding of specific or compatible lesions in the brains of aborted foetuses as described by others [11] and the identification of at least one specific immunodominant antigen (IDA) either in the foetal fluids or sera by an immunoblotting technique. There were no paired samples in this study i.e. the negative and positive reference samples from aborted foetuses did not come from their corresponding aborted cows.

The sera from the aborted foetuses and aborting breeding cattle were collected within a month of abortion.

Group 3: Positive $(n=22)$ and negative reference sera $(n=27)$ from heifers and cows without any recent individual abortion problem belonging to herds with a previous diagnosis of Neospora abortion as described previously. All samples were analysed by immunoblotting and positive sera detected at least one IDA.

Group 4: The target population was composed of infected and non-infected heifers and cows $(n=372)$ from 11 herds with a previous history of Neospora abortion problems in the last three years. An individual diagnosis of the infection using immunoblotting was also carried out.

After blood recovery, all sera and foetal fluids used in this study were aliquoted and stored at $-80^{\circ} \mathrm{C}$ prior to testing for specific antibodies against $N$. caninum.

Secondly, two different age groups were analysed (foetuses and breeding cattle belonging to groups 1 and 2) in order to investigate the distribution of their serologic values and justify the use of different cut-off points for the IFAT and ELISA according to the age of the animal.

Thirdly, cut-off points were calculated for IFAT and ELISA according to the purpose of testing. In order to diagnose abortion, the cut-off points were estimated for foetuses and breeding cattle (groups 1 and 2). A cut-off point to diagnose infection was also calculated (group 3).
Finally, the cut-off points obtained to diagnose infection were revalidated. In this way, the cut-off points obtained for group 3 were applied to a target population (group 4) and their effect on the tests performance characteristics and prevalence estimates were investigated.

\subsection{Parasite and antigen preparation}

$N$. caninum tachyzoites ( $\mathrm{Nc}-1$ isolate) were obtained by continuous passage in Vero cell culture following previously described standard procedures [19]. The parasites were harvested from tissue culture and washed three times in sterile $0.3 \mathrm{M}$ PBS, $\mathrm{pH} 7.4$ and separated from host cell debris by passaging the mixture through a 25-gauge needle, following a passage through a $5 \mu \mathrm{m}$ polycarbonate filter. $\mathrm{Nc}-1$ purified tachyzoites were pelleted and stored at $-80{ }^{\circ} \mathrm{C}$ until use. Tachyzoites for IFAT were resuspended in phosphatebuffered saline (PBS) to a final concentration of approximately $10^{7} / \mathrm{mL}$. Soluble antigen extract, used in western blot, was prepared as follows: to obtain $N$. caninum soluble proteins, purified tachyzoites $(2 \times$ $10^{9}$ ) were suspended in $1 \mathrm{~mL}$ of $10 \mathrm{mM}$ Tris hydrochloride containing $2 \mathrm{mM}$ of phenylmethylsulfonyl fluoride (Sigma Chemical Co., St. Louis, Mo, USA), disrupted by ultrasonic treatment (Branson mod. Sonifier 450, Branson Ultrasonic Co., USA) in an ice-bath, and centrifuged at $10000 \times g$ for $20 \mathrm{~min}$ at $4{ }^{\circ} \mathrm{C}$. Protein content was determined using the Micro BCA protein assay method (Pierce, Rockford, USA) [29], and the supernatant was aliquoted and cryopreserved at $-80{ }^{\circ} \mathrm{C}$.

\subsection{IFAT technique}

The procedure was carried out basically as described by others [33]. The cattle sera were diluted at two-fold serial dilutions starting at a 1:25 dilution in PBS to the end point titre. Unbroken tachyzoite membrane fluorescence was considered as a positive reaction. 


\subsection{ELISA technique}

A crude antigen ELISA (Civtest ${ }^{\circledR}$ Hipra Laboratories S.A., Gerona, Spain) [24], was run as recommended by the manufacturer and positive and negative controls were provided with the kit. The test results were expressed as O.D. values.

\subsection{SDS-PAGE and western blot analysis}

Electrophoresis was performed according to a previous work [18] in $12.5 \%$ polyacrylamide gels. Low molecular weight standards (Bio-Rad Laboratories; California, USA) were subjected to electrophoresis concurrently so that the rates of migration $(\mathrm{Mr})$ of the different antigens recognised by the sera could be estimated. Proteins were electrophoretically transferred to a nitrocellulose membrane for western blot (Mini Trans-Blot Cell). The cow sera and foetus fluid dilutions corresponded to 1:100 and 1:50 respectively. As a secondary antibody, a mouse monoclonal anti-bovine antibody IgG1 and IgG2 (1:200) (Hipra Laboratories S.A.; Gerona, Spain) was used and antigen-antibody reactions were developed using 4-chloro1naphtol (Bio-Rad Laboratories; California, USA) as the substrate. Intense recognition of at least one of the following 4 immunodominant proteins $(17-18,34$ 35,37 and $60-62 \mathrm{kDa}$ ) by aborted and infected cows as well as by foetuses was regarded as a positive result [1].

\subsection{Analysis of data}

The non-parametric Mann Whitney $U$-test was employed to investigate the distribution of serologic values in both bovine populations.

ROC analysis was applied to estimate cut-off points by employing two different softwares, AccuROC for Windows 95/98/ NT 2.0 and TG-ROC CMDT [12]. The areas under the ROC curve (AUC) together with the standard error of the AUC $\left(\mathrm{SE}_{\mathrm{AUC}}\right)$ were calculated to compare the overall diagnostic performances of both IFAT and ELISA [13]. The cut-off points obtained by ROC analysis using AccuROC were selected for either maximal diagnostic sensitivity or maximal diagnostic specificity values for IFAT and ELISA and in the absence of a cut-off point for both maximal sensitivity and specificity an interval of unclear results was established. The TG-ROC CMDT approach was employed to confirm the selection of different ELISA cut-off points $\left(d_{o}\right)$ for equal sensitivity and specificity $\left(\theta_{o}\right)$ for the different groups. A non-parametric approach for correlated samples was employed to compare the AUC [9] for IFAT and ELISA for the different groups considered. True prevalence values obtained in a target population for the different cut-off points considered for infected cattle were compared by a contingency tables analysis. Diagnostic sensitivity and specificity were recalculated by ROC analysis and apparent prevalence, negative (NPV) and positive predictive values (PPV) were also estimated and considered in the true prevalence calculation $[16,31]$.

The STAT-VIEW v.4.0. package (Abacus Concepts, Inc. Berkeley, California, USA) was used to calculate the contingency tables and Mann Whitney $U$-test analysis.

The precision of both serological techniques was also measured by their repeatability including interassay and operatorto-operator variations [16], which were calculated in triplicate for seven positive samples and seven negative samples.

\section{RESULTS}

\subsection{Distribution of serologic values in different bovine populations}

No significant differences were found between the heifers and cows in group 1 when tested by IFAT and ELISA. When negative reference sera from foetuses and 
Table I. IFAT and ELISA values for reference sera sample populations of Neospora infected and non-infected bovine foetuses and breeding cattle.

\begin{tabular}{|c|c|c|c|c|c|c|c|c|}
\hline & & \multicolumn{3}{|c|}{ Foetuses } & \multicolumn{3}{|c|}{ Heifers-cows } & \multirow[t]{2}{*}{$P$ value } \\
\hline & & Median & $\begin{array}{c}\text { Upper } \\
\text { Quartile }\end{array}$ & $\begin{array}{l}\text { Lower } \\
\text { Quartile }\end{array}$ & Median & $\begin{array}{c}\text { Upper } \\
\text { Quartile }\end{array}$ & $\begin{array}{l}\text { Lower } \\
\text { Quartile }\end{array}$ & \\
\hline \multirow{2}{*}{ IFAT* } & Negative & 0 & 0 & 0 & 0 & 0 & 0 & 0.2755 \\
\hline & Positive & $1: 128$ & $1: 500$ & $1: 32$ & $1: 500$ & $1: 1000$ & $1: 250$ & 0.0012 \\
\hline \multirow{2}{*}{ ELISA** } & Negative & 0.077 & 0.082 & 0.072 & 0.111 & 0.137 & 0.095 & 0.0001 \\
\hline & Positive & 0.728 & 0.907 & 0.102 & 1.060 & 1.642 & 0.805 & 0.0008 \\
\hline
\end{tabular}

$P$ value based on the Mann-Whitney rank test.* IFAT titres. ** O.D. values.

breeding cattle (heifers and cows) were processed by the IFAT technique, no significant differences were found since none of the samples showed positive IFAT titres from a starting 1:25 dilution. However, significant differences were found when these two groups of samples were processed by the ELISA (Tab. I). For group 2, heifers and cows (breeding cattle) also had different distribution of serologic values from foetuses when positive reference samples from both bovine populations were tested by both techniques.

\subsection{Determination of cut-off values}

The cut-off values obtained by ROC analysis (Fig. 1) for IFAT and ELISA with reference sera from either aborted cows and foetuses belonging to groups 1 and 2 or infected cattle from group 3 are summarised in Table II. In aborted breeding cattle the cut-off point obtained by ROC analysis for IFAT and ELISA gave 100\% diagnostic sensitivity and specificity as well as for IFAT in the case of the foetuses. In infected breeding cattle for both techniques and in foetuses for ELISA, two possible cut-off values were obtained, one for a maximum sensitivity and one for a maximum specificity (Tab. II). When equal sensitivity and specificity values were considered following the TG-ROC CMDT approach, we also obtained different ELISA cut-off values $\left(d_{o}\right)$ for the different groups ( 0.381 for aborting breeding cattle,
0.373 for infected breeding cattle and 0.093 for foetuses) with a $\theta_{o}$ value equal or higher than 0.9 .

\subsection{ROC curve comparison}

Comparison of the ROC curves for correlated samples obtained with IFAT and ELISA for the different groups considered demonstrated a negligible difference in the overall accuracy of both tests. For aborted breeding cattle and foetuses from groups 1 and 2, the AUC values obtained were as follows: $\mathrm{AUC}_{\mathrm{IFAT}}=1 ; \mathrm{AUC}_{\mathrm{ELISA}}=1$, and $\mathrm{AUC}_{\mathrm{IFAT}}=1 ; \mathrm{AUC}_{\mathrm{ELISA}}=0.99$, respectively. Significant differences were only found for AUC in infected breeding cattle from group $3(P<0.05)$ with an AUC of 0.9 and 1 for IFAT and ELISA, respectively. In all cases, their corresponding standard error values were always smaller than 0.05 .

\subsection{Diagnostic characteristics and prevalence as a function of the cut-off point}

Prevalence and diagnostic characteristics for the different cut-off points considered for infected breeding cattle are summarised in Table III. Significant differences in the diagnostic characteristics depending on the cut-off point and technique considered were observed. The cutoff points with better diagnostic values and 

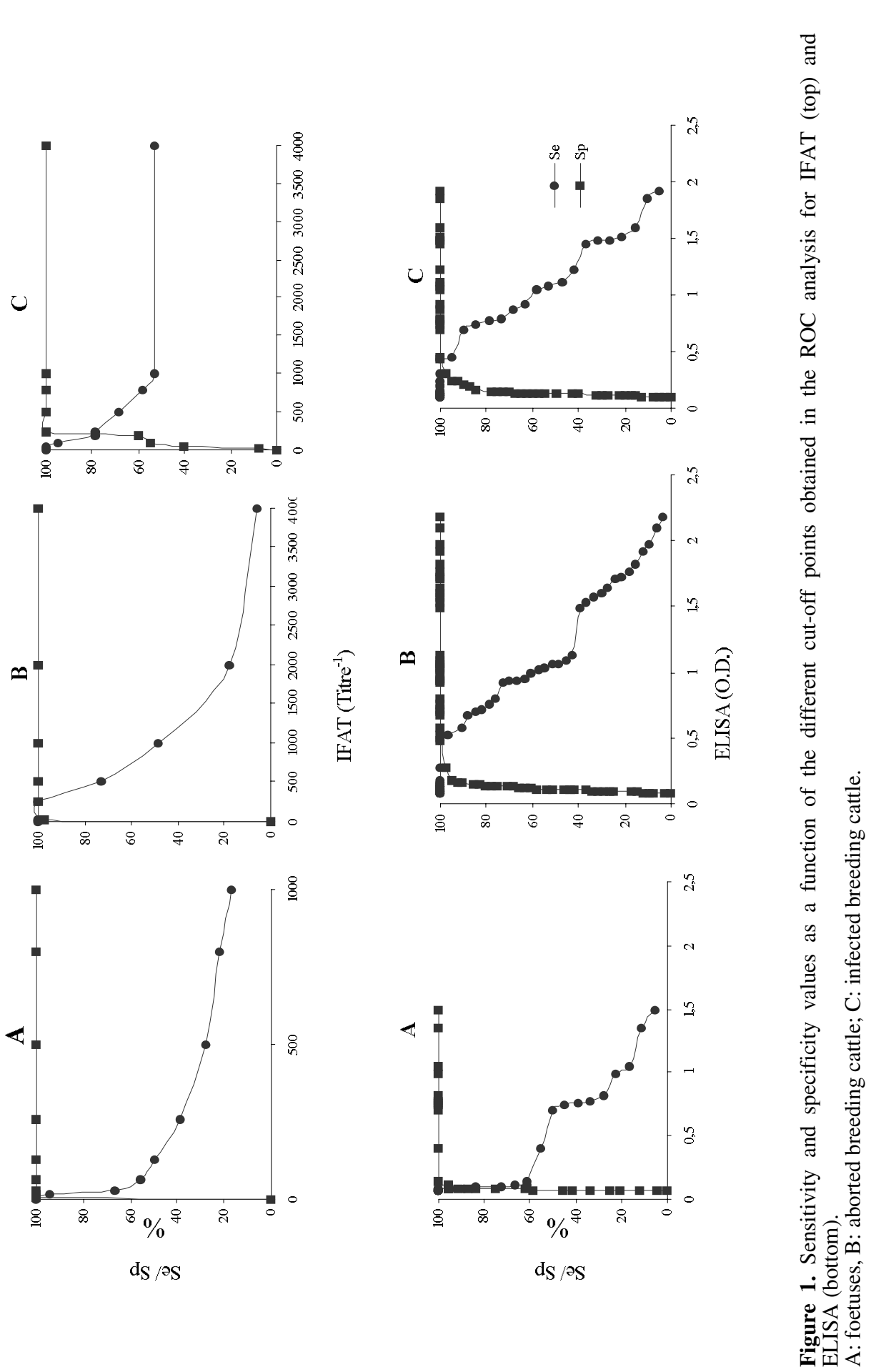
Table II. Cut-off values suggested by ROC analysis for IFAT and ELISA as a function of animal age and technique purpose.

\begin{tabular}{lccc}
\hline & $\begin{array}{c}\text { Aborted } \\
\text { foetuse }\end{array}$ & \multicolumn{2}{c}{ Breeding cattle } \\
\cline { 3 - 4 } & & Aborted cattle Infected cattle \\
\hline IFAT $^{*}$ & $1: 16^{\mathrm{a}, \mathrm{b}}$ & $1: 250^{\mathrm{a}, \mathrm{b}}$ & $1: 100^{\mathrm{a}}$ \\
& & & $1: 250^{\mathrm{b}}$ \\
ELISA $^{* *}$ & $0.098^{\mathrm{a}}$ & $0.484^{\mathrm{a}, \mathrm{b}}$ & $0.306^{\mathrm{a}}$ \\
& $0.401^{\mathrm{b}}$ & & $0.451^{\mathrm{b}}$ \\
& $0.093^{\mathrm{c}}$ & $0.381^{\mathrm{c}}$ & $0.373^{\mathrm{c}}$ \\
\hline
\end{tabular}

a Cut-off values for a maximum sensitivity. ${ }^{\mathrm{b}}$ Cutoff values for a maximum specificity. ${ }^{\mathrm{c}}$ Cut-off values for equal sensitivity and specificity $(\theta>$ 0.90). * IFAT titres; ${ }^{* *}$ O.D. values.

similar apparent and true prevalence results corresponded to the cut-off points estimated in this work, 1:250 for IFAT and 0.306-0.451 for ELISA.

When true prevalence values were compared, significant differences were obtained for 1:100 and 1:250, as well as for 1:250 and 1:640 IFAT cut-off points $(P<0.05)$. The differences observed in the true prevalences for the ELISA cut-off points turned out to be non-significant despite the lower prevalence obtained when the cut-off point suggested by the manufacturer was considered. When true prevalence rates corresponding to IFAT and ELISA cut-off points for maximum sensitivity were compared, significant differences were obtained $(P<0.01)$, whereas for maximum specificity the differences observed were not significant.

\subsection{Precision of IFAT and ELISA}

As shown in Table IV, most of the coefficients of variation for ELISA and IFAT were lower than $10 \%$.

\section{DISCUSSION}

A few attempts have been made to compare and critically evaluate the different serological methods currently in use for bovine neosporosis. It has been recommended that several issues, such as appropriate selection of the reference population and sampling strategies, should be taken

Table III. IFAT and ELISA diagnostic characteristics and prevalences obtained in a target population according to the different cut-off points considered for infected breeding cattle.

\begin{tabular}{lccccccc}
\hline Technique & $\begin{array}{c}\text { Cut-off } \\
\text { values }\end{array}$ & Se & Sp & PPV & NPV & $\begin{array}{c}\text { Apparent } \\
\text { prevalence }\end{array}$ & $\begin{array}{c}\text { True } \\
\text { prevalence }\end{array}$ \\
\hline IFAT & $1: 100^{\mathrm{a}}$ & 95 & 55 & 15.1 & 99.4 & 48.9 & 7.8 \\
(titre) & $1: 250^{\mathrm{b}}$ & 78.9 & 100 & 100 & 92 & 23.1 & 29.2 \\
& $1: 640^{\mathrm{c}}$ & 57.8 & 100 & 100 & 92 & 8.8 & 16.4 \\
\hline ELISA & $0.306^{\mathrm{a}}$ (O.D.) & 100 & 97.5 & 91.3 & 100 & 24.7 & 22.7 \\
& $0.451^{\mathrm{b}}$ (O.D.) & 94.7 & 100 & 100 & 98 & 23.9 & 25.2 \\
& $5^{\mathrm{c}}$ (IRCP)* & 100 & 85 & 53 & 100 & 27.6 & 14.8 \\
\hline
\end{tabular}

Se: Diagnostic sensitivity. Sp: Diagnostic specificity. PPV: Positive predictive value. NPV: Negative predictive value. IRCP: Relative index $\times 100$.

${ }^{\text {a }}$ Cut-off value selected for a maximum sensitivity. ${ }^{\mathrm{b}}$ Cut-off value selected for a maximum specificity.

${ }^{c}$ Cut-off value suggested in the literature for IFAT [6] and ELISA [24].

$$
* \text { IRCP }=\frac{\left(\text { O.D. }{ }_{405} \mathrm{~S}-\text { O.D. }{ }_{405} \mathrm{NC}\right)}{\text { (O.D. } \left.{ }_{405} \mathrm{PC}-\text { O.D. }{ }_{405} \mathrm{NC}\right)} \times 100 \quad \begin{aligned}
& \mathrm{S}=\text { sample, } \mathrm{NC}=\text { mean negative control, } \mathrm{PC}= \\
& \text { mean positive control. }
\end{aligned}
$$


Table IV. Interassay and operator-to-operator precision of ELISA and IFAT.

\begin{tabular}{|c|c|c|c|c|c|c|c|c|}
\hline \multirow[t]{3}{*}{ Sample No. } & \multicolumn{4}{|c|}{ ELISA (O.D.) \# } & \multicolumn{4}{|c|}{ IFAT titre $\left(\log _{2}\right) \#$} \\
\hline & \multicolumn{2}{|c|}{ Mean } & \multicolumn{2}{|c|}{$\% \mathrm{CV}^{*}$} & \multicolumn{2}{|c|}{ Mean } & \multicolumn{2}{|c|}{$\% \mathrm{CV}^{*}$} \\
\hline & $\mathrm{a}$ & $\mathrm{b}$ & $\mathrm{a}$ & $\mathrm{b}$ & a & $\mathrm{b}$ & $\mathrm{a}$ & $\mathrm{b}$ \\
\hline \multicolumn{9}{|l|}{$\begin{array}{l}\text { Positive } \\
\text { samples }\end{array}$} \\
\hline 1 & 1.729 & 1.618 & 4.0 & 6.3 & 10.23 & 10.23 & 5.6 & 5.6 \\
\hline 2 & 1.741 & 1.756 & 1.3 & 6.6 & 10.63 & 10.63 & 5.4 & 5.4 \\
\hline 3 & 1.612 & 1.651 & 3.3 & 5.3 & 9.97 & 9.30 & 0.0 & 6.2 \\
\hline 4 & 0.909 & 0.898 & 12.3 & 2.5 & 9.30 & 9.97 & 6.2 & 0.0 \\
\hline 5 & 1.718 & 1.719 & 3.4 & 5.7 & 10.30 & 9.30 & 5.6 & 6.2 \\
\hline 6 & 1.061 & 1.039 & 9.4 & 13.9 & 10.63 & 10.63 & 5.4 & 5.4 \\
\hline 7 & 2.004 & 1.907 & 9.0 & 5.8 & 11.97 & 10.97 & 0.0 & 9.1 \\
\hline \multicolumn{9}{|l|}{$\begin{array}{l}\text { Negative } \\
\text { animals }\end{array}$} \\
\hline 1 & 0.115 & 0.119 & 1.0 & 8.6 & 6.64 & 6.31 & 0.0 & 9.1 \\
\hline 2 & 0.100 & 0.097 & 10.2 & 14.9 & 6.31 & 5.98 & 9.1 & 9.7 \\
\hline 3 & 0.123 & 0.125 & 3.7 & 5.6 & 6.64 & 5.31 & 0.0 & 10.9 \\
\hline 4 & 0.129 & 0.130 & 5.1 & 8.0 & 5.64 & 4.98 & 0.0 & 11.6 \\
\hline 5 & 0.104 & 0.117 & 11.5 & 9.0 & 5.98 & 4.98 & 9.7 & 11.6 \\
\hline 6 & 0.122 & 0.130 & 4.2 & 8.7 & 5.98 & 4.98 & 9.7 & 11.6 \\
\hline 7 & 0.134 & 0.125 & 6.0 & 2.0 & 5.64 & 4.98 & 17.7 & 11.6 \\
\hline
\end{tabular}

\# The given values are the means of three independent measures. * CV, coefficient of variation. ${ }^{\text {a Val- }}$ ues for interassay precision. ${ }^{b}$ Values for operator-to-operator precision.

into consideration when validating a veterinary diagnostic test. Diagnostic sensitivity and specificity are test performance parameters that could be highly influenced by factors such as stage of disease and animal age [13]. In the present work, we applied a validation procedure, as proposed recently $[13,16,17]$, to IFAT and an indirect ELISA, two of the techniques most used world-wide for the diagnosis of neosporosis in cattle. Therefore, restrictive criteria for selection of the reference population, selection of the cut-off point as a function of animal age and disease status were considered as crucial steps in the validation process. The combination of multi- ple tests, such as epidemiological, clinical, histopathological and serological measures, is considered as a gold standard to define the reference populations used in our study [16]. Three main approaches have been previously followed to establish the reference populations: the serological status of the new-born calf as an indicator of the dam status assuming a high efficiency of vertical transmission [8], the simultaneous use of two serological techniques to confirm the status of the animal [27] and the use of the IFAT test as a gold standard $[4,25]$. In our opinion, the first approach introduces a source of error mainly due to a not very high efficiency of 
vertical transmission observed in natural conditions in some endemically infected herds - from $48 \%$ [21] to 95\% [8] - and the fact that experimentally infected model animals infected before pregnancy, which showed consistent specific antibody titres during gestation, did not transmit the infection to their descendants [15]. On the contrary, the use of IFAT as a gold standard does not appear to be accurate enough in a validation study since there is still some uncertainty concerning standard cutoff values either for adults or foetuses, since cut-off titres in IFAT differ between laboratories and are often set at 1:160 $1: 640[4]$ or $1: 25$ to $1: 640[6,28]$ for adult bovines and from 1:25 to $1: 80$ for foetal serology [3, 5, 35]. Besides, the IFAT test cannot resolve non-specific or suspicious positive reactions, whereas western blot is more specific [30] and allows unequivocal serological diagnosis even in cases that are problematic for IFAT testing.

One of the most important biological factors that could affect the distribution of the test value, as well as the diagnostic sensitivity and specificity, is animal age [13]. Bovine foetuses are only able to develop a serologically detectable response from month $4-5$ of gestation onwards [20]. On the contrary, specific antibody titres observed in the 7-12 month-old calves and in heifers [21] or seroprevalence in the 13 to 24 month-age group [7] are lower than in other age groups, probably related to the decline in maternal antibodies in congenitally infected cattle [34]. Although they are not surprising, our results confirm the use of different cut-off points for foetuses and breeding cattle, and the homogeneity of serologic values for a $N$. caninum infected breeding cattle population composed of heifers and cows.

With regards to the use of the tests, different approaches have been followed. Whereas the traditional calculation of cutoff points has been followed by most authors, recently a new approach based on Bayes theorem was reported for the sero- logic diagnosis of Neospora infection [32]. In the present work, the calculation of cutoff points was carried out depending on the intended uses of the tests. Therefore a combination of ROC analysis and utility-based decision theory was followed to calculate the cut-off points for the two main independent bovine populations defined in this study: foetuses and breeding cattle and for two purposes of testing. The cut-off points obtained for Neospora aborted foetuses and Neospora aborted breeding cattle corresponds to an improvement in serology to diagnose the cause of abortion, since cutoff points for both techniques were obtained following the same criteria and using a well characterised panel of reference sera. This approach permits more precise cut-off points to be defined for a desired sensitivity and specificity according to the different practical applications for which a serological test is supposed to be used. In both cases, lower IFAT cut-off points were obtained either for maximal sensitivity or specificity compared to those described by others [4, 28] without an increment of false positive results. On the contrary, the cut-off values obtained for aborted dams were higher than for infected animals in agreement with previously described higher antibody titres in Neospora-aborted cattle compared with seropositive non-aborted cattle [21]. In this case, cut-off points estimated for by two different ROC analyses were very similar and confirmed the necessity of using different cut-off points. TG-ROC CMDT software determines a cut-off point for equal sensitivity and specificity values, which might not correspond to any of the cut-off points obtained in the practical field, whereas the AccuROC software provides sensitivity and specificity values for the different cut-off points obtained in the experiment.

From a practical viewpoint and in the absence of a perfect cut-off point for infected breeding cattle, the use of a value for maximum sensitivity could be 
interesting to investigate the status of individual cattle prior to purchase or entry into a Neospora-free herd. On the contrary, a cut-off for maximum specificity could be of use when evaluating the presence of the disease in an area. A final possibility could be the use of both cut-off points obtained by the AccuROC approach or the cut-off point obtained by TG-ROC CMDT assuming an equivalent cost of false positive and negative results. This decision implies the use of a confirmatory test for intermediate results [2], which could be western blot based on the identification of a/some tachyzoite immunodominant antigens [1, 27] by the problematic sera.

The agreement between IFAT and ELISA has been studied using the kappa index [23, 26], and moderate and good results have been obtained in different studies. On the contrary, different ELISAs have also been compared by the kappa index [36] and by correlation analysis using paired results [27] obtaining results with good agreement and high coefficients of determination, respectively. In the present work, the comparison of ROC curves suggests the possibility of using IFAT or ELISA interchangeably. The area under the ROC curve (AUC) has been suggested as a global statistic summary of the overall performance of a test [14], and the high AUC values for both tests indicated a good positive-negative classification. This assumption is confirmed by the similar negative and positive predictive values and similar true prevalence results obtained in the prevalence study carried out in a target population with a cut-off point of 1:250 for IFAT and 0.306 or 0.451 for ELISA. However, when a greater diagnostic sensitivity is required, ELISA offers more accurate diagnostic characteristic values compared to the 1:100 IFAT cut-off point.

\section{ACKNOWLEDGEMENTS}

We would like to thank Vanessa Navarro and Beatriz Peinado for their excellent technical assistance. We also thank the Regional Veterinary Laboratories from Lugo (Galicia) and Derio (Basque Country) for providing some of the samples used in this study. This work has been supported by a research grant from the Spanish government (CICYT-AGF98-0804C02) and Gema Álvarez García was financed by Consejería de Educación (Comunidad de Madrid).

\section{REFERENCES}

[1] Álvarez García G., Pereira Bueno J., Gómez Bautista M., Ortega Mora L.M., Pattern of recognition of Neospora caninum tachyzoite antigens by naturally infected pregnant cattle and aborted foetuses, Vet. Parasitol. 107 (2002) 17-27.

[2] Atkinson R., Harper P.A.W., Reichel M.P., Ellis J.T., Progress in the serodiagnosis of Neospora caninum infections of cattle, Parasitol. Today 16 (2000) 110-114.

[3] Barr B.C., Anderson M.L., Sverlow K.W., Conrad P.A., Diagnosis of bovine fetal Neospora infection with an indirect fluorescent antibody test, Vet. Rec. 137 (1995) 611613.

[4] Björkman C., Uggla A., Serological diagnosis of Neospora caninum infection, Int. J. Parasitol. 29 (1999) 1497-1507.

[5] Buxton D., Caldow G., Maley S., Marks J., Innes E.A., Neosporosis and bovine abortion in Scotland, Vet. Rec. 141 (1997) 649-651.

[6] Conrad P.A., Sverlow K., Anderson M., Rowe J., BonDurant R., Tuter G., Breitmeyer R., Palmer C., Thurmond M., Ardans A., Dubey J.P., Duhamel G., Barr B., Detection of serum antibody responses in cattle with natural or experimental Neospora infections, J. Vet. Diagn. Invest. 5 (1993) 572-578.

[7] Davison H.C., French N.P., Trees A.J., Herdspecific and age specific seroprevalence of Neospora caninum in 14 British dairy herds, Vet. Rec. 144 (1999) 547-550.

[8] Davison H.C., Greiner M., Trees A.J., Quantitative analysis of Neospora caninum serological data obtained from dairy cattle. Proceedings of the Society of Veterinary Epidemiology and Preventive Medicine, Annual Conference, Bristol, UK, 1999, pp. 172-181.

[9] DeLong E.R., DeLong D.M., Clarke-Pearson D.L., Comparing the areas under two or more 
correlated receiver operating characteristics curves: A nonparametric approach, Biometrics 44 (1988) 837-845.

[10] Dubey J.P., Recent advances in Neospora and neosporosis, Vet. Parasitol. 84 (1999) 349-367.

[11] González L., Buxton D., Atxaerandio R., Aduriz G., Maley S., Marco J.C., Cuervo L.A., Bovine abortion associated with Neospora caninum in Northern Spain, Vet. Rec. 144 (1999) 145-150.

[12] Greiner M., Two-graph receiver operating characteristic (TG-ROC) - a Microsoft-Excel template for the selection of cut-off values in diagnostic tests, J. Immunol. Methods 185 (1995) 145-146.

[13] Greiner M., Gardner I.A., Epidemiologic issues in the validation of veterinary diagnostic tests, Prev. Vet. Med. 45 (2000) 3-22.

[14] Greiner M., Pfeiffer D., Smith R.D., Principles and practical application of the receiveroperating characteristic analysis for diagnostic tests, Prev. Vet. Med. 45 (2000) 23-41.

[15] Innes E.A., Wright S.E., Maley S., Rae A., Schock A., Kirvar E., Bartley P., Hamilton C., Carey I.M., Buxton D., Protection against vertical transmission in bovine neosporosis, Int. J. Parasitol. 31 (2001) 1523-1534.

[16] Jacobson R.H., Validation of serological assays for diagnosis of infectious diseases, Rev. Sci. Tech. O.I.E. 17 (1998) 507-526.

[17] Jenkins M., Baszler T., Björkman C., Schares G., Williams D., Diagnosis and seroepidemiology of Neospora caninum-associated bovine abortion, Int. J. Parasitol. 32 (2002) 631-636.

[18] Laemmli U.K., Cleavage of structural proteins during the assembly of the head of bacteriophage T4, Nature 227 (1970) 680685.

[19] Lindsay D.S., Dubey J.P., In vitro development of Neospora caninum (Protozoa: Apicomplexa) from dogs, J. Parasitol. 75 (1989) 163-165.

[20] Osburn B.I., Ontogeny of immune responses in cattle, in: The ruminant immune system in health and disease, Morrison I, 2nd ed., Cambridge University Press, Cambridge, Great Britain, 1988, pp. 252-260.

[21] Pereira-Bueno J., Quintanilla-Gozalo A., Seijas-Carballedo A., Costas E., Ortega-Mora L.M., Observational studies in Neospora caninum infected dairy cattle: pattern of transmission and age-related antibody fluctuations, in: Hemphill A., Gottstein B.
(Eds.), A European perspective on Neospora caninum, Int. J. Parasitol. 30 (2000) 906-909.

[22] Quintanilla-Gozalo A., Pereira-Bueno J., Seijas-Carballedo A., Costas E., Ortega-Mora L.M., Observational studies in Neospora caninum infected dairy cattle: relationship infection-abortion and gestational antibody fluctuations, in: Hemphill A., Gottstein B. (Eds.), A European perspective on Neospora caninum, Int. J. Parasitol. 30 (2000) 900-906.

[23] Quintanilla-Gozalo A., Pereira-Bueno J., Tabarés E., Innes E.A., González-Paniello R., Ortega-Mora L.M., Seroprevalence of Neospora caninum infection in dairy and beef cattle in Spain, Int. J. Parasitol. 29 (1999) 1201-1208.

[24] Rebordosa X., Álvarez-García G., Collantes E., Ortega L.M., Artigas C., Desarrollo de un ELISA indirecto para la valoración de anticuerpos contra Neospora caninum, Laboratorio Veterinario, Avedila 17 (2000) 5-8.

[25] Reichel M.P., Pfeiffer D.U., An analysis of the performance characteristics of serological tests for the diagnosis of Neospora caninum infection in cattle, Vet. Parasitol. 107 (2002) 197-207.

[26] Schares G., Peters M., Wurn W., Barwald A., Conraths F.J., The efficiency of vertical transmission of Neospora caninum in dairy cattle analysed by serological techniques, Vet. Parasitol. 80 (1998) 87-98.

[27] Schares G., Conraths F.J., Reichel M.P., Bovine neosporosis: comparison of serological methods using outbreak sera from a dairy herd in New Zealand, Int. J. Parasitol. 29 (1999) 1659-1667.

[28] Schares G., Rauser M., Zimmer K., Peters M., Wurm R., Dubey J.P., de Graaf D.C., Edelhofer R., Mertens C., Hess G., Conraths F.J., Serological differences in Neospora caninum-associated epidemic and endemic abortions, J. Parasitol. 85 (1999) 688-694.

[29] Smith R.D., Krohn R.I., Hermanson G.T., Mallia A.K., Gartner F.H., Provenzano M.D., Fujimoto E.K., Goeke N.M., Olson B.J., Klenj D.C., Measurement of protein using bicinchoninic acid, Anal. Biochem. 150 (1985) 76-85

[30] Söndgen P., Peters M., Bärwald A., Wurm R., Holling F., Conraths F.J., Schares G., Bovine neosporosis: immunoblot improves foetal serology, Vet. Parasitol. 102 (2001) 279-290 
[31] Thrusfield M., Diagnostic testing, in: Blackwell Science Ltd (Ed.), Veterinary Epidemiology, The University Press, Cambridge, Great Britain, 1995, pp. 266-285.

[32] Thurmond M.C., Johnson W.O., MuñozZanzi C.A., Su C.L., Hietala S.K., A method of probability diagnostic assignment that applies Bayes theorem for use in serologic diagnostics, using an example of Neospora caninum infection in cattle, Am. J. Vet. Res. 63 (2002) 318-325.

[33] Trees A.J., Guy F., Tennant B.J., Balfour A.H., Dubey J.P., Prevalence of antibodies to Neospora caninum in a population of urban dogs in England, Vet. Rec. 132 (1993) 125126.
[34] Williams D.J.L., Guy F., Garry J.W., Guy F., Tasker L., Smith R.F., MacEachem K., Cripps P.J., Kelly D.F., Trees A.J., Neospora caninum associated abortion in cattle: the time of experimentally-induced parasitaemia during gestation determines foetal survival, Parasitology 121 (2000) 347-358.

[35] Wouda W., Dubey J.P., Jenkins M.C., Serological diagnosis of bovine foetal neosporosis, J. Parasitol. 83 (1997) 545-547.

[36] Wouda W., Brinkhof J., van Maanen C., de Gee A.L., Moen A.R., Serodiagnosis of neosporosis in individual cows and dairy herds, a comparative study of three enzymelinked immunosorbent assays, Clin. Diagn. Lab. Immunol. 5 (1998) 711-716. 